\title{
Preparation of a new superhydrophobic/ superoleophilic corn straw fiber used as an oil absorbent for selective absorption of oil from water
}

\author{
Yang $\mathrm{Xu}^{1 \dagger}$, Haiyue Yang ${ }^{1 \dagger}$, Deli Zang ${ }^{1}$, Yan Zhou ${ }^{4}$, Feng Liu' ${ }^{1}$, Xiaochen Huang ${ }^{2}$, Jo-Shu Chang ${ }^{2,3}$,
} Chengyu Wang ${ }^{1 *}$ and Shih-Hsin $\mathrm{Ho}^{2^{*}}$

\begin{abstract}
Background: Oil leakages frequently occur during oil product development and oil transportation. These incidents are a vital factor in water contamination, thus leading to serious environmental destruction. Therefore, superhydrophobic/superoleophilic material is one of the solutions to treat oily wastewater.

Results: This study aimed to develop a simple, fast and low-cost method to treat oily wastewater by synthesizing a new superhydrophobic/superoleophilic corn straw fiber via conventional impregnation. The corresponding results illustrate that abundant homogeneous silica $\left(\mathrm{SiO}_{2}\right)$ granules evenly accreted on the surface of the prepared fiber were conducive to high surface roughness. Meanwhile, (Heptadecafluoro-1,1,2,2-tetradecyl) trimethoxysilane, a sort of silane coupling agent, could greatly reduce surface free energy by grafting with $\mathrm{SiO}_{2}$ particles on the corn straw fiber surface. The obtained superhydrophobic/superoleophilic corn straw fiber exhibited a water contact angle of $152^{\circ}$ and an oil contact angle of $0^{\circ}$ for various oils, strongly demonstrating its considerable application as an oil absorbent that can be applied for oil cleanup. In addition, the prepared fiber displayed a great chemical stability and environmental durability. Conclusions: Due to its high absorption capacity and absorption efficiency, the prepared fiber has great potential as a new oil absorbent for treatment of oily water.
\end{abstract}

Keywords: Oil absorption, Corn straw, Superhydrophobic, Superoleophilicity, $\mathrm{SiO}_{2}$ particles

\section{Background}

Oil-spill pollution can significantly destroy the human living environment and poses a severe threat to ecological systems (Cojocaru et al. 2011; Deng et al. 2013; Schaum et al. 2010). At present, physical methods

\footnotetext{
*Correspondence: wangcy@nefu.edu.cn; stephen6949@msn.com; stephen6949@hit.edu.cn

${ }^{\dagger}$ Yang Xu and Haiyue Yang contributed equally to this work and share first authorship

${ }^{1}$ Key Laboratory of Bio-based Material Science and Technology, Ministry of Education, Northeast Forestry University, Harbin 150040, People's Republic of China

${ }^{2}$ State Key Laboratory of Urban Water Resource and Environment, School of Environment, Harbin Institute of Technology, Harbin 150090, People's Republic of China

Full list of author information is available at the end of the article
}

(Angelova et al. 2011; Howarter and Youngblood 2007), chemical processing methods and biological treatment (Boopathy et al. 2012) to cleanup oil-bearing wastewater have been reported. Among them, physical adsorption techniques, which employ hydrophilic materials to remove waste oil from water, is considered as the most economic and valid method due to its high absorption capacity and low cost. However, most of the traditional oil absorbents generally encounter problems, such as low separation efficiency, high cost, poor absorption capacity and non-biodegradable characteristics (Karakasi and Moutsatsou 2010). Thus, fabrication of new environmental-friendly oil absorbing material with a higher absorption ability and lower production cost is required and urgent (Yao et al. 2011). Using waste biomass materials as 
an oil absorbent is of great interest for oil-water separation and is becoming an area of intense research because they are cheap, accessible, and biodegradable (Vlaev et al. 2011; Zang et al. 2016).

Recently, superhydrophobic surfaces have attracted attention because of their unique properties, including self-cleaning, anti-sticking, water proof, chemical stability, and oil recovery (Yuan et al. 2013; Zang et al. 2015). In theory, construction of a hierarchical rough structure with low surface energy are two indispensable parameters for the formation of superhydrophobic surfaces, which are characterized by a water contact angle greater than $150^{\circ}$ and a water sliding angle less than $10^{\circ}$ (Kim et al. 2011; Li et al. 2010; Wang et al. 2011a). To date, multiple technologies and various materials have been proposed to achieve superhydrophobic and superoleophilic surfaces, such as the solution-immersion process, chemical etching, layer-by-layer assembly, laser treating, and ultrasound irradiation (Liang and Guo 2013; Zhang et al. 2013; Zhou et al. 2013).

Corn straw fiber, a natural and biodegradable biomass from agricultural waste, is usually discarded and burned on the spot, thus resulting in severe air pollution. In fact, efficient utilization of straw resources is vital to solving the serious air pollution caused by straw burning. Zang et al. (2016) removed oil from water using superhydrophobic/superoleophilic corn straw fibers that they prepared with $\mathrm{ZnO}$ particles via conventional impregnation. $\mathrm{ZnO}$ particles are hollow spheres with an average diameter approximately $5 \mu \mathrm{m}$, and a hexadecyltrimethoxysilane (HDTMOS) chemical agent is employed to act as a hydrophobic modifier. In this study, we exploited corn straw fibers as a raw material to prepare a high-efficiency oil absorbent that exhibits great chemical stability and environmental friendliness. The superhydrophobic/superoleophilic properties of the prepared corn straw fiber arose from the combined effects from the deposition of homogeneous $\mathrm{SiO}_{2}$ inorganic particles, with an average particle size about 40-50 nm using the sol-gel method, and the hydrophobic embellishment of (Heptadecafluoro-1,1,2,2-tetradecyl) trimethoxysilane (PTES), thus giving the product the ability to efficiently dislodge oils from oily wastewater. Compared to HDTMOS, the amount of PTES is less; therefore, the production cost is reduced. In view of its intrinsic water-repellence, high absorption capacity, chemical stability and environmental friendliness, the prepared corn straw fiber floats on the surface of the water after absorbing the oil, allowing it to be easily transported and recycled. The information obtained in this study demonstrates that the prepared superhydrophobic/superoleophilic corn straw fiber can be widely applied in the treatment of oily wastewater, thereby providing new insight into producing a sustainable high-efficiency oil absorbent from agricultural waste.

\section{Methods \\ Materials and chemicals}

Corn straw from a farm in Harbin Jiangbei District was used in this study. Ethanol (99.7\%), sodium hydroxide (96.0\%), hydrogen peroxide $(30.0 \%)$, hydrochloric acid (37\%), ammonium hydroxide (25.0\%), tetraethoxysilane (98.0\%) and glacial acetic acid (99.5\%) were purchased from Tianjin Kemiou Chemical Reagent Co., Ltd. (Tianjin, China). (Heptadecafluoro-1,1,2,2-tetradecyl) trimethoxysilane (99.5\%), employed to decorate $\mathrm{SiO}_{2}$ granules, was purchased from Aladdin Chemicals Co., Ltd. (Shanghai, China). All chemicals were used as received without any purification. Diesel oil, gasoline, crude oil, bean oil, n-hexane, octane, toluene, and chloroform, supplied by Sigma-Aldrich Co., Ltd. (St. Louis, MO, USA), were used for oil contact angle testing and absorption capacity measurements.

\section{Pre-treatment of the corn straw fiber}

Peeled corn straw was placed in a pulveriser to obtain straw fibers, and then sieved through 60 and 80 mesh standard screens to collect fibers $(250-425 \mu \mathrm{m})$. In addition, corn straw fibers were ultrasonically rinsed three times with deionized water, ethanol and deionized water. The corn straw was then placed in an aqueous solution of $\mathrm{NaOH}(100 \mathrm{~mL}, 0.5 \mathrm{wt} \%)$ and $30 \% \mathrm{H}_{2} \mathrm{O}_{2}(3.5 \mathrm{~mL})$ with stirring at ambient temperature for $14 \mathrm{~h}$. This helped in removing the resins and additional impurities present in the corn straw and exposed the hydroxy groups. Next, the $\mathrm{pH}$ of above solution was adjusted to 6.5-7.0 with $\mathrm{HCl}(6 \mathrm{~mol} / \mathrm{L})$. After washing several times with deionized water to remove chemical residues, the pretreated corn straw was dried at $40{ }^{\circ} \mathrm{C}$ for $24 \mathrm{~h}$ until its weigh remained constant.

\section{Synthesis of $\mathrm{SiO}_{2}$ particles}

The $\mathrm{SiO}_{2}$ particles were fabricated by a sol-gel process. Briefly, $10 \mathrm{~mL} \mathrm{NH} \mathrm{NH}_{3} \mathrm{OH}$ was added dropwise into a beaker containing $180 \mathrm{~mL}$ ethanol, $20 \mathrm{~mL}$ tetraethoxysilane and $20 \mathrm{~mL}$ deionized water. The solution was vigorously stirred for $2 \mathrm{~h}$ at room temperature. Next, the mixture was left for $10 \mathrm{~h}$ to produce a white suspension. Homogeneous $\mathrm{SiO}_{2}$ particles were dissociated from the white suspension by centrifugation in ethanol and then dried at $60{ }^{\circ} \mathrm{C}$ for $6 \mathrm{~h}$.

\section{Fabrication of superhydrophobic/superoleophilic corn straw fiber}

In detail, $0.1 \mathrm{~g} \mathrm{SiO}_{2}$ was added to a solution of $10 \mathrm{~mL}$ anhydrous ethanol, $0.03 \mathrm{~mL}$ PTES, $0.025 \mathrm{~mL} \mathrm{H}_{2} \mathrm{O}$, and $0.005 \mathrm{~mL}$ 
acetic acid. Subsequently, $0.2 \mathrm{~g}$ corn straw was added into the mixture. The reaction was maintained under stirring at room temperature for $5 \mathrm{~h}$, and then placed in an oven at $65^{\circ} \mathrm{C}$ for $3 \mathrm{~h}$. Finally, the superhydrophobic/superoleophilic corn straw fibers were acquired by drying the corn straw at $50^{\circ} \mathrm{C}$ until its weight was consistent.

In this study, the PTES chemical agent acted as a hydrophobic modifier, with its modification mechanism as follows (Fig. 1): silicon hydroxyl groups, which were generated from a hydrolysis reaction with the PTES reagent, reacted with hydroxyl groups on the surfaces of $\mathrm{SiO}_{2}$ particles and pristine corn straw fibers. Therefore, the hydrophobic heptadecafluoro-decylalkyl of PTES was introduced onto the surface of the fibers to induce the low surface energy of superhydrophobic/superoleophilic corn straw.

\section{Characterization of pristine corn straw fiber} and superhydrophobic/superoleophilic corn straw fiber Scanning electron microscope (SEM) images were collected on a Hitachi TM3030 tabletop microscope. The chemical composition of the prepared corn straw product was detected by Fourier transform infrared spectroscopy (FTIR, Magna-IR 560, Nicolet, USA), X-ray photoelectron spectroscopy (XPS, PHI Thermo Fisher Scientific, USA) and energy-dispersive $\mathrm{X}$-ray analysis (EDX, Quantax70, USA). Water contact angle (WCA) and oil contact angle (OCA) measurements were carried out on a contact angle instrument (CA-A, Hitachi, Japan) by dropping a $5 \mu \mathrm{L}$ deionized water or oil onto five or more different positions on the corn straw specimens. The values of the WCA and OCA were determined as averages of those of five measurements.

\section{Evaluation of absorption capability and oil removal efficiency}

An absorption capability test was performed in pure oil by suspending a nylon net bag containing $0.5 \mathrm{~g}$ corn straw into a beaker with $150 \mathrm{~mL}$ oil at room temperature. After $5 \mathrm{~h}$, the nylon bag was taken out from the oil and let to stand for $10 \mathrm{~min}$. Absorption capability calculated using Eq. (1):

$$
q=\left(m_{2}-m_{1}\right) / m_{1}
$$

where $q$ is the absorption capability $(\mathrm{g} / \mathrm{g}) ; m_{2}$ is the weight of corn straw fibers after the absorption; and $m_{1}$ is the initial weight of corn straw fibers prior to absorption.

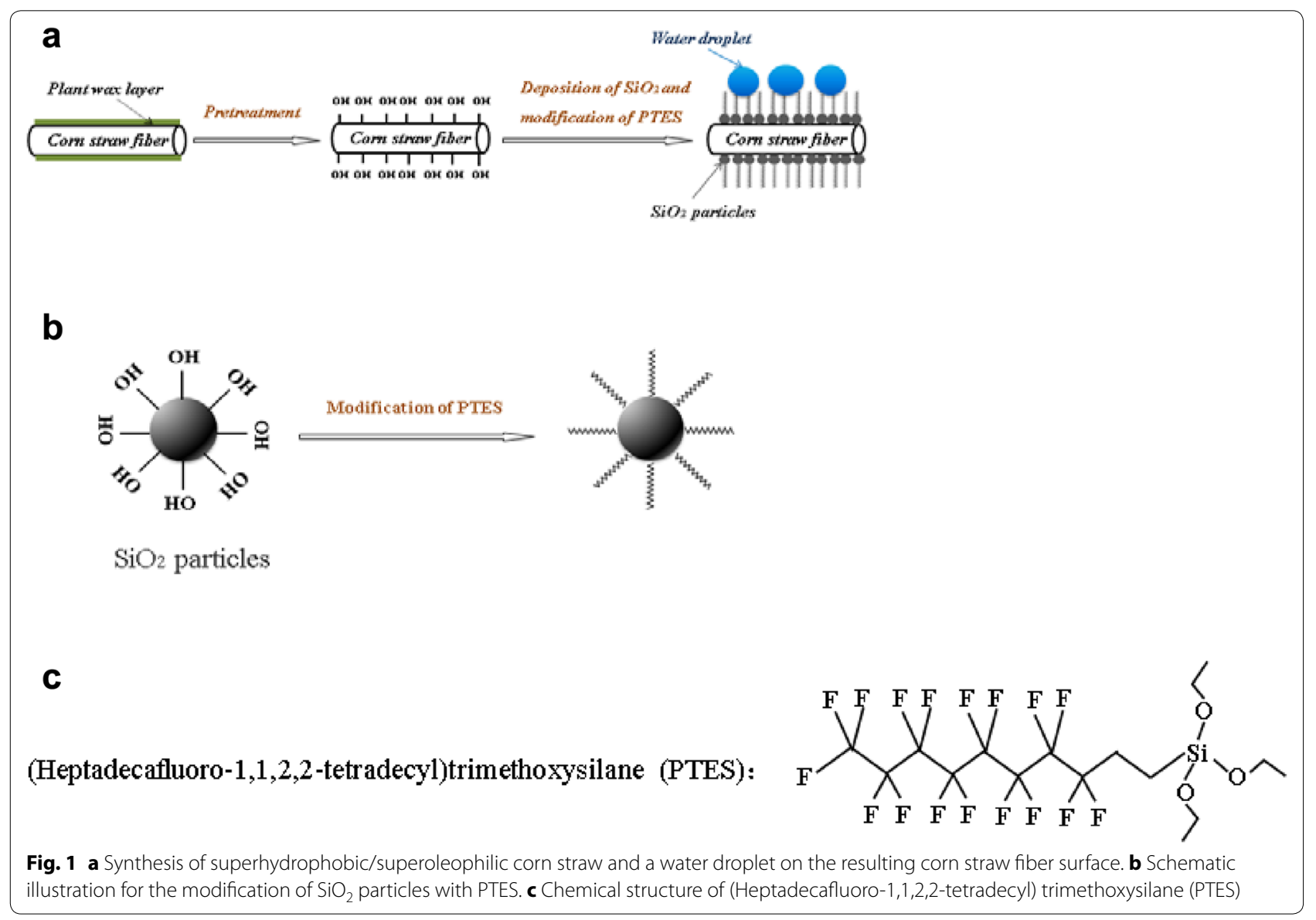


Oil removal efficiency experiments were performed in an oil-water system, which was similar to the oil absorption capability test. Nylon net bags containing $0.5 \mathrm{~g}$ corn straw were placed in $150 \mathrm{~mL}$ oil-water mixtures with different mass ratios, and then stirred $(500 \mathrm{r} / \mathrm{min})$ at room temperature. After $5 \mathrm{~h}$, the nylon bags were removed from the oil and let to stand for $10 \mathrm{~min}$. The oil removal efficiency was calculated using Eq. (2):

$$
k=\left(w_{3}-w_{2}-w_{1}\right) /\left(w_{3}-w_{2}\right)
$$

where $k$ is the oil removal efficiency (\%); $w_{3}$ is the weight of the corn straw fibers after absorption; $w_{2}$ is the initial weight of the corn straw fibers prior to absorption; and $w_{1}$ is the weight of water absorbed in the absorbents.

In this study, one-way analysis of variance (AVONA) was used to analyze the reliability of the experimental data. All experimental data are presented as averages of five measurements. A $p$ value $\geq 0.95$ for the Student's $t$ test demonstrates reliability of the experimental data.

\section{Results and discussion}

Micro-structure analysis of a novel superhydrophobic/ superoleophilic corn straw fiber

It is known that surface microtopography is primarily responsible for establishing superhydrophobic surface, similar to the lotus leaf's self-cleaning property that is associated with its micro/nanostructure (Wang et al. 2015; Autumn et al. 2000; Ju et al. 2012; Wei et al. 2010; Gao et al. 2009; Zhang et al. 2008). Therefore, it is crucial to survey the surface micro-profiles of corn straw fiber by SEM. The corresponding surface morphology results of raw corn straw fiber and superhydrophobic/superoleophilic corn straw fiber are shown in Fig. 2. At low SEM magnifications (Fig. 2a, b), there was no marked difference in the fibrous shape between untreated sample and
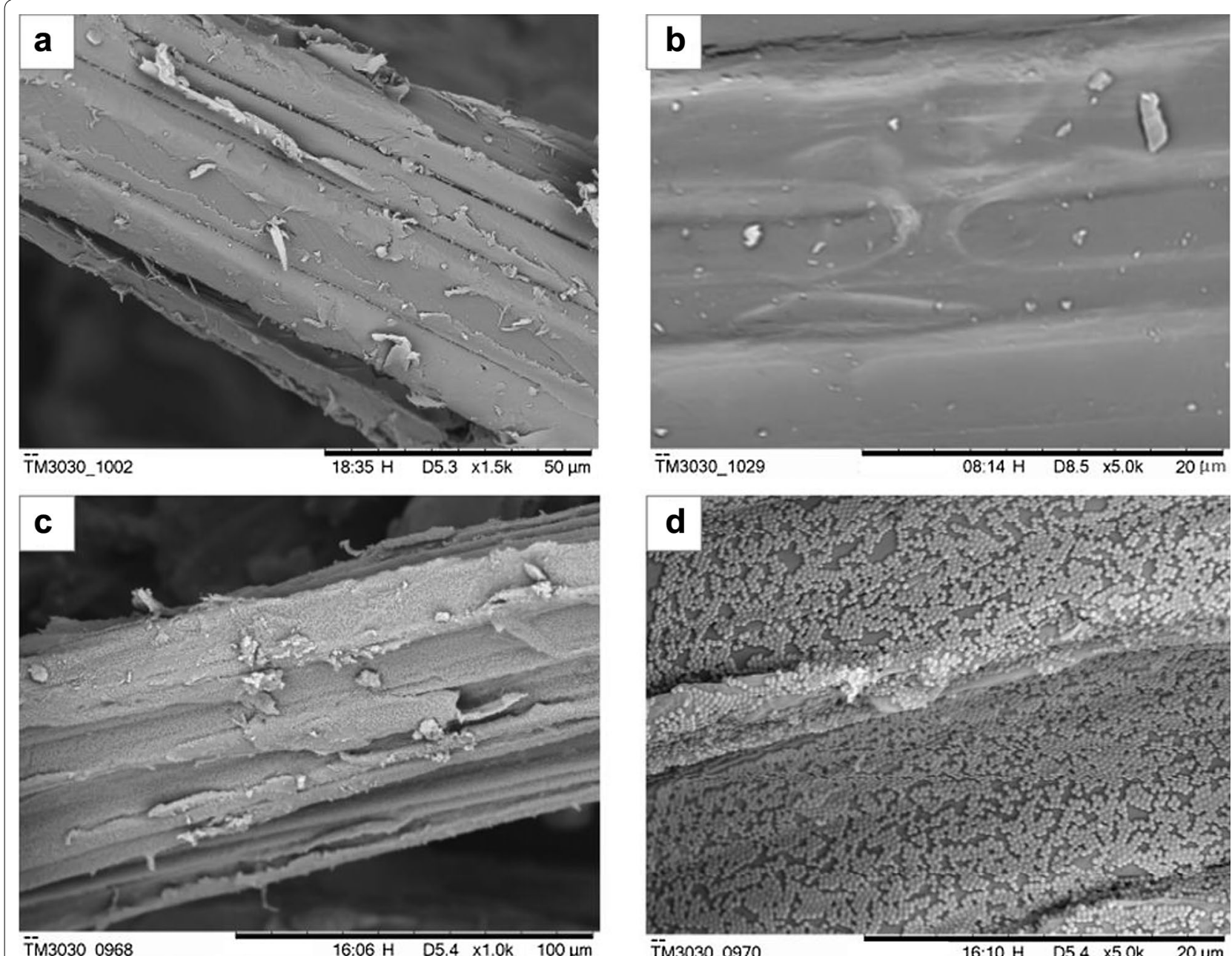

Fig. 2 SEM images of the raw corn straw fiber $(\mathbf{a}, \mathbf{b})$ and superhydrophobic/superoleophilic corn straw fiber $(\mathbf{c}, \mathbf{d})$ at different magnifications. Compared to raw corn straw fiber, $\mathrm{SiO}_{2}$ particles compactly deposit on the superhydrophobic/superoleophilic corn straw fiber surface 
modified product, indicating that the product retained the characteristics of the original corn straw fiber. In contrast with the smooth fiber surface of pristine corn straw, the high magnification SEM image of the prepared superhydrophobic/superoleophilic corn straw fiber showed a surface layer of solid spherical granules, with an average diameter of 40-50 nm (Fig. 2c), which was attributed to the compact deposition of the $\mathrm{SiO}_{2}$ particles.

\section{Surface wettability of superhydrophobic/superoleophilic corn straw fiber}

The principle of surface wettability can usually be demonstrated by the Young equation, as follows (Wang et al. 2015):

$$
\cos \theta=\frac{\gamma_{\mathrm{sv}}-\gamma_{\mathrm{sl}}}{\gamma_{\mathrm{lv}}}
$$

where $\gamma_{\mathrm{sv}}, \gamma_{\mathrm{sl}}$ and $\gamma_{\mathrm{lv}}$ are the interfacial free energy of solid/vapor, solid/liquid, and liquid/vapor, respectively; and $\theta$ is the contact angle. In general, the value of the contact angle is an essential to measuring the surface wettability of a superhydrophobic material. Hence, the water/oil contact angles of corn straw fiber were investigated to determine its superhydrophobic/superoleophilic characteristics. For raw corn straw fiber, a water contact angle of $0^{\circ}$ was visible on fiber surface, which is ascribed to massive hydroxyl groups on a pristine fiber surface (Fig. 3a). In contrast, a spherical water droplet was observed on the prepared fiber, with a water contact angle of $152^{\circ}$ (Fig. 3c), indicating its superior superhydrophobicity. Moreover, when dripped on the surface of the raw corn straw fiber and prepared corn straw fiber, oil droplets instantly spread, indicating an oil contact angle of $0^{\circ}$ (Fig. 3b, d). Uniform coverage of sub-micrometer $\mathrm{SiO}_{2}$ microspheres, coupled with micron-sized corn straw fiber, while using the PTES function as a modifier to ornament $\mathrm{SiO}_{2}$ particles would facilitate low surface energy. The combination of a particularly hierarchical rough structure and low surface energy is regarded as an indispensable condition when constructing special superhydrophobic and superoleophilic material from the resulting corn straw fiber surface. Because air was captured and trapped, while falling onto corn straw fiber surface, by the abundant cavities and interspaces among $\mathrm{SiO}_{2}$ particles on the fiber surface, a water droplet could contact the trapped air to manifest a non-wetting phenomenon. As soon as water was dropped on the prepared corn straw fiber surface, it was repelled without leaving a
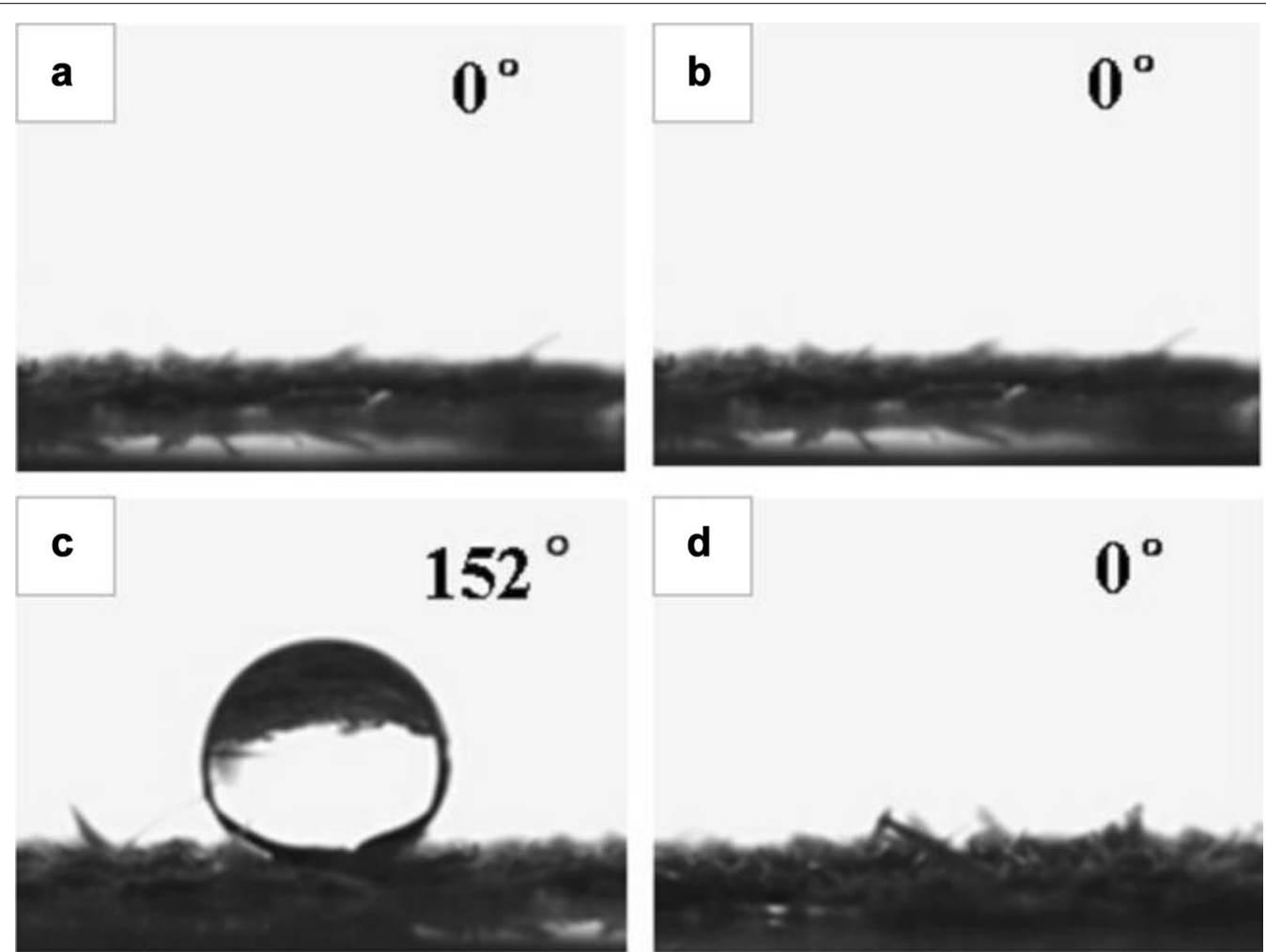

Fig. 3 Images of a water droplet (a) and an oil droplet on raw corn straw fiber (b); a water droplet (c) and an oil droplet (d) on prepared superhydrophobic/superoleophilic corn straw fiber. Compared to raw corn straw fiber, the resulting corn straw fiber indicates its superior superhydrophobicity 
trail, which demonstrates the great waterproof characteristic of the resulting product (Wang et al. 2011b). Accordingly, it could be deduced that the wettability of the corn straw fiber was transformed from superhydrophilicity to superhydrophobicity. Taken together, these results demonstrate the superhydrophobic and superoleophilic properties of the prepared corn straw fiber.

In this study, the combination of numerous $\mathrm{SiO}_{2}$ particle aggregates and surface modification by PTES could prevent water from wetting the treated fiber surface and result in water droplets on the obtained corn straw fiber surface rolling off without leaving a trace, thereby demonstrating a novel non-wetting material. Hence, the prepared superhydrophobic/superoleophilic corn straw fiber absorbs only oil while completely repelling water.

\section{Surface chemical component analysis}

In this study, $\mathrm{SiO}_{2}$ particles were prepared using the Stöber method, where tetraethoxysilane and ammonium hydroxide acted as a precursor and a catalyst, respectively. The synthesis process of $\mathrm{SiO}_{2}$ particles was divided into two stages, which included the hydrolysis of tetraethoxysilane and condensation polymerization of the hydrolyzed intermediate in the presence of the ammonia catalyst. The concrete forming process of the $\mathrm{SiO}_{2}$ particles was as follows (Wang et al. 2011b):

(1)Hydrolysis:

$$
\begin{aligned}
& \mathrm{Si}-\left[\mathrm{OC}_{2} \mathrm{H}_{5}\right]_{4}+4 \mathrm{H}_{2} \mathrm{O} \\
& \quad \rightarrow \mathrm{Si}-(\mathrm{OH})_{4}+4 \mathrm{C}_{2} \mathrm{H}_{5} \mathrm{OH} .
\end{aligned}
$$

(2) Alcohol condensation:

$$
\begin{aligned}
& \mathrm{Si}-(\mathrm{OH})_{4}+\mathrm{Si}-\left[\mathrm{OC}_{2} \mathrm{H}_{5}\right]_{4} \\
& \quad \rightarrow \equiv \mathrm{Si}-\mathrm{O}-\mathrm{Si} \equiv+4 \mathrm{C}_{2} \mathrm{H}_{5} \mathrm{OH} .
\end{aligned}
$$

(3) Water condensation:

$$
\begin{aligned}
& \mathrm{Si}-(\mathrm{OH})_{4}+\mathrm{Si}-(\mathrm{OH})_{4} \\
& \quad \rightarrow \equiv \mathrm{Si}-\mathrm{O}-\mathrm{Si} \equiv+4 \mathrm{H}_{2} \mathrm{O} .
\end{aligned}
$$

Large amounts of hydroxyl groups on the surface of the silica particles are critical for the preparation of superhydrophobic/superoleophilic corn straw fibers (Wang et al. 2011b). In addition, due to the great influence of silica size distribution on the generation of a superhydrophobic surface, we strictly abided to the well-known Stöber method for fabrication of silica particles.

FTIR, XPS and EDX were used to analyze the surface chemical composition of superhydrophobic/ superoleophilic corn straw fiber. The relevant FTIR spectra of $\mathrm{SiO}_{2}$ particles, pristine corn straw fiber and superhydrophobic/superoleophilic corn straw fiber are listed in Fig. 4. The absorption peak at $955 \mathrm{~cm}^{-1}$ was ascribed to stretching vibration of isolated $\mathrm{Si}-\mathrm{OH}$, which was perceptible only in the case of bare silica (Fig. 4a) (Kulkarni et al. 2008). Moreover, corresponding to Si-O$\mathrm{Si}$ asymmetric stretching and symmetric stretching, the bands at 1056 and $795 \mathrm{~cm}^{-1}$ were also observable (Hsieh et al. 2010; Vinogradova et al. 2006). Compared with pristine corn straw fiber, the band at $804 \mathrm{~cm}^{-1}$ was due to $\mathrm{Si}-\mathrm{O}-\mathrm{Si}$ symmetric stretching (Hsieh et al. 2010) and the absorption peak at $1203 \mathrm{~cm}^{-1}$ was a typical characteristic of the C-F stretching vibration of PTES (Zhou et al. 2013), which proves that the $\mathrm{SiO}_{2}$ particle deposition and PTES organic chemistry reagent were observed on the prepared superhydrophobic/superoleophilic corn straw fiber surface (Fig. 4b).
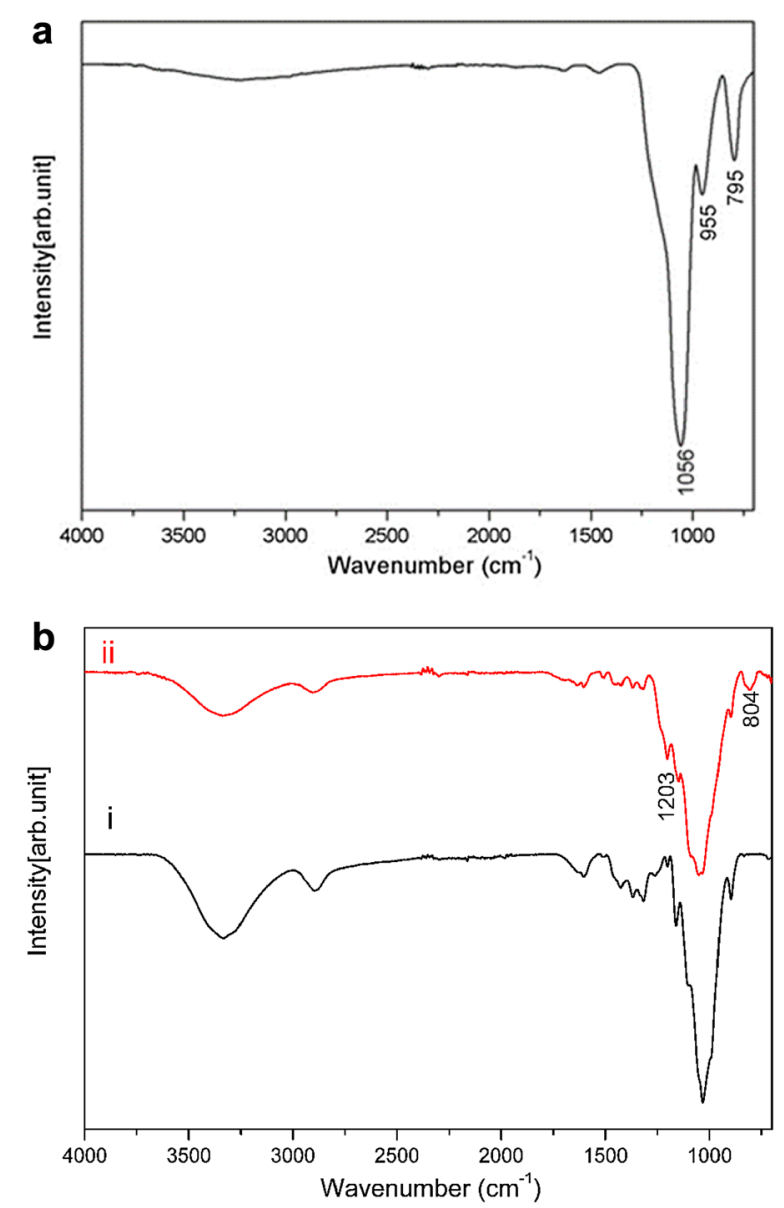

Fig. 4 a FTIR spectra of $\mathrm{SiO}_{2}$ particles; $\mathbf{b}$ FTIR spectra of raw corn straw fiber (i) and prepared superhydrophobic/superoleophilic corn straw fiber (ii) 
The XPS spectra of pristine corn straw fiber and superhydrophobic/superoleophilic corn straw fiber are shown in Fig. 5. With regards to raw corn straw fiber (Fig. 5a), only peaks corresponding to $\mathrm{C} 1 \mathrm{~s}$ and $\mathrm{O} 1 \mathrm{~s}$ elements were

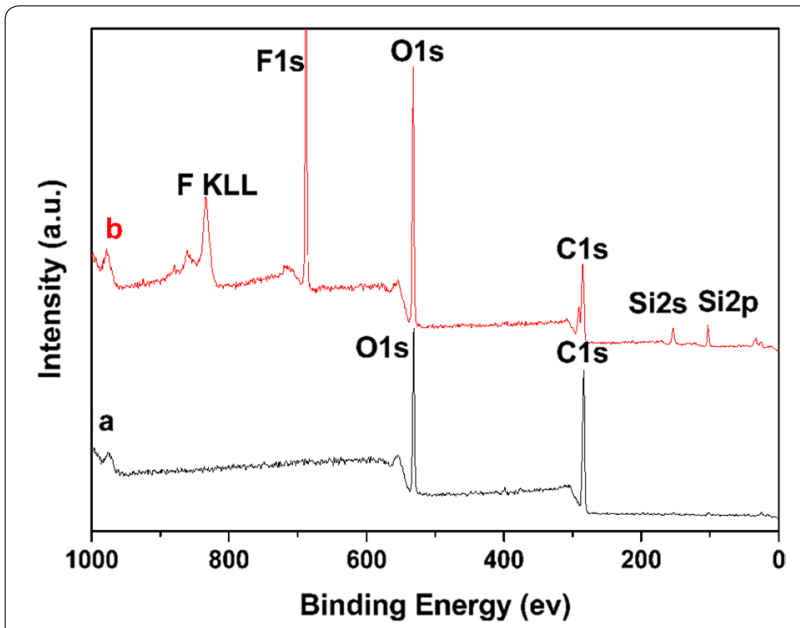

Fig. 5 XPS spectra of pristine corn straw fiber (a) and superhydrophobic/superoleophilic corn straw fiber (b) observed. By contrast, the XPS spectra of superhydrophobic/superoleophilic corn straw fiber demonstrated four new peaks including Si2p, Si2s, F1s and F KLL, which accounted for the generation of $\mathrm{SiO}_{2}$ particles and PTES on the prepared fiber surface. However, the peak intensity of $\mathrm{C} 1 \mathrm{~s}$ and $\mathrm{O} 1 \mathrm{~s}$ in curve $b$ was weaker than that in curve $a$. This can be attributed to the addition of $\mathrm{SiO} 2$ particles and PTES, thereby decreasing the relative mass ratio of $\mathrm{C} 1 \mathrm{~s}$ and $\mathrm{O} 1 \mathrm{~s}$.

Apart from FTIR and XPS characterizations, the elemental composition of superhydrophobic/superoleophilic corn straw fiber was investigated via energydispersive X-ray analysis (EDX). The oxygen $(\mathrm{O})$ peak and the carbon $(C)$ peak were observed in corn straw fiber (Fig. 6). In comparison with the raw fiber, there were two new peaks of silica $(\mathrm{Si})$ and fluorine $(\mathrm{F})$ induced by $\mathrm{SiO}_{2}$ and PTES in the prepared superhydrophobic/superoleophilic corn straw fiber, thus providing evidence for the presence of $\mathrm{SiO}_{2}$ particles and PTES on the obtained corn straw fiber surface. Taken together, these results show that $\mathrm{SiO}_{2}$ particles were successfully modified by PTES and truly existed on the surface of the superhydrophobic/superoleophilic corn straw fiber.

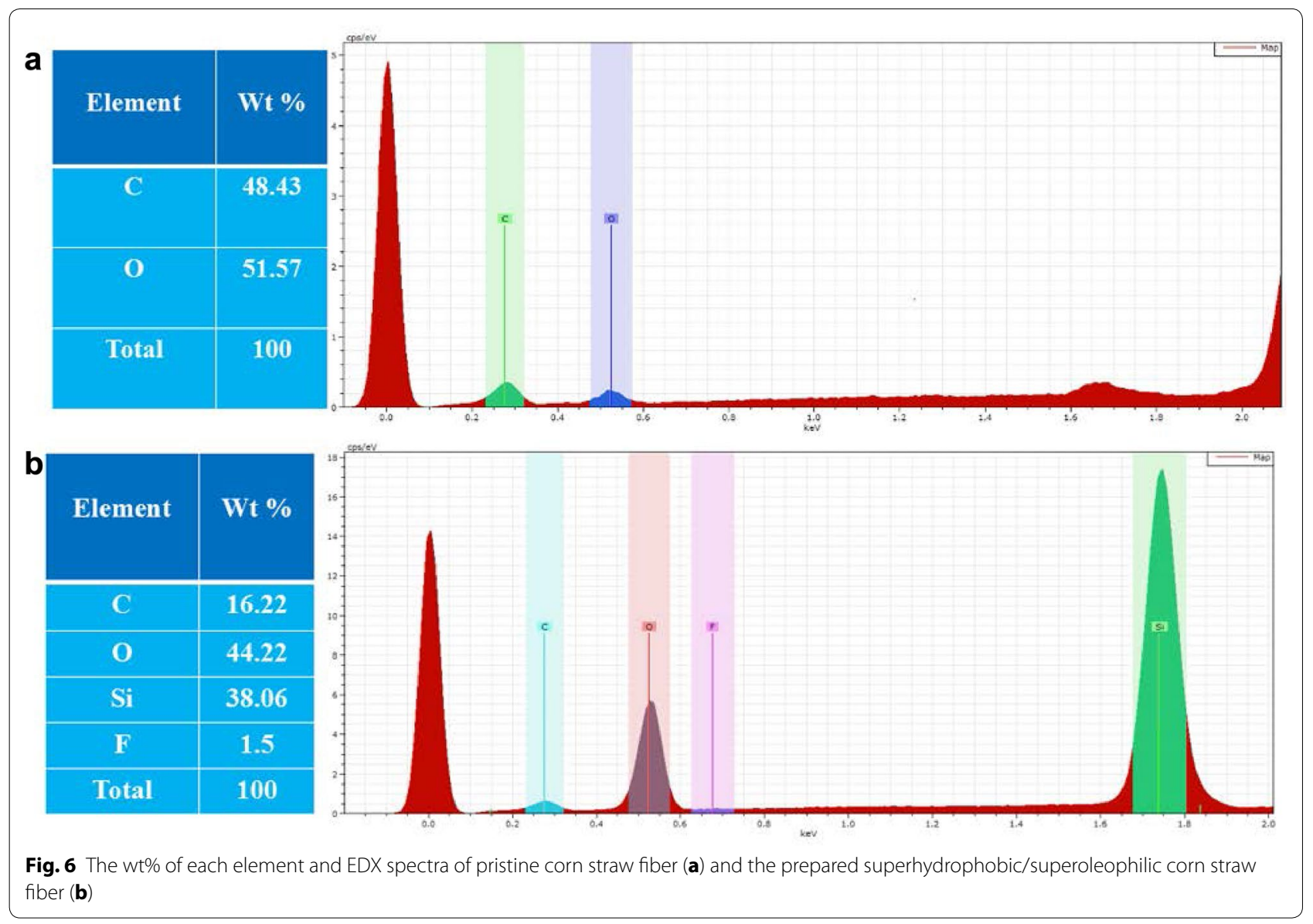




\section{Environmental durability and application in water-oil separation}

Considering the importance of environment durability and chemical stability for prepared materials used in practical application, it is necessary to investigate these properties with respect to superhydrophobic/superoleophilic corn straw fiber to confirm its potential as an oil absorbent. The effects of acidic and alkaline conditions on the wettability of superhydrophobic/superoleophilic corn straw fiber were systematically investigated. Contact angle measurements were performed by pipetting $5 \mu \mathrm{L}$ aqueous solution, from $\mathrm{pH} 0-14$, onto fiber surfaces in order to evaluate chemical stability and durability of the prepared material (Fig. 7a). The measured water contact angle ranged from $152^{\circ}$ to $150^{\circ}$, while the oil contact angle remained constant at $0^{\circ}$, implying that the resulting fiber surface still maintained outstanding superhydrophobicity and superoleophilicity properties, even in strong acid and strong alkaline conditions. To evaluate its environmental stability, the contact angles of water and
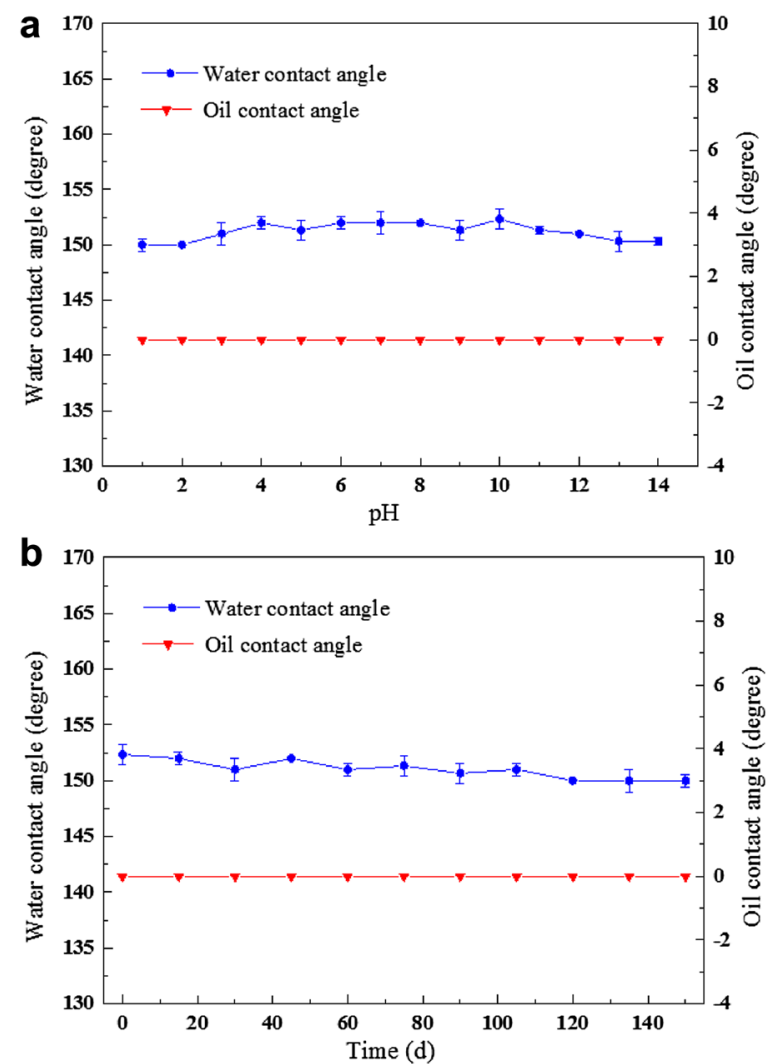

Fig. 7 a Variation of water contact angle and oil contact angle of superhydrophobic/superoleophilic corn straw surfaces in aqueous solutions with different $\mathrm{pH}$ values. $\mathbf{b}$ The relationship between contact angles of the resulting superhydrophobic/superoleophilic corn straw fibers and days of storage in air environment oil of the superhydrophobic/superoleophilic corn straw fiber were monitored over time at ambient temperature and humidity (Fig. 7b). After 150 days, there were no distinct changes in the water and oil contact angles of the prepared corn straw fiber, which clearly demonstrates that the superhydrophobic/superoleophilic corn straw fiber obtained in this study possess excellent environment stability.

Because the prepared corn straw exhibited favorable superhydrophobic/superoleophilic performance in both acidic solutions and under ambient conditions, these prepared fibers could be utilized as highly selective absorption materials to achieve effective separation of oil-water mixtures. Oil absorption performance is measured by the absorption capacity $(\mathrm{g} / \mathrm{g})$, as well as the oil removal efficiency (\%). To determine the maximum absorption capacity of superhydrophobic/superoleophilic corn straw fiber, experiments were performed in a variety of pure oils and organic solvents. Figure 8 a presents the absorption capacities of raw corn straw fiber, pretreated corn
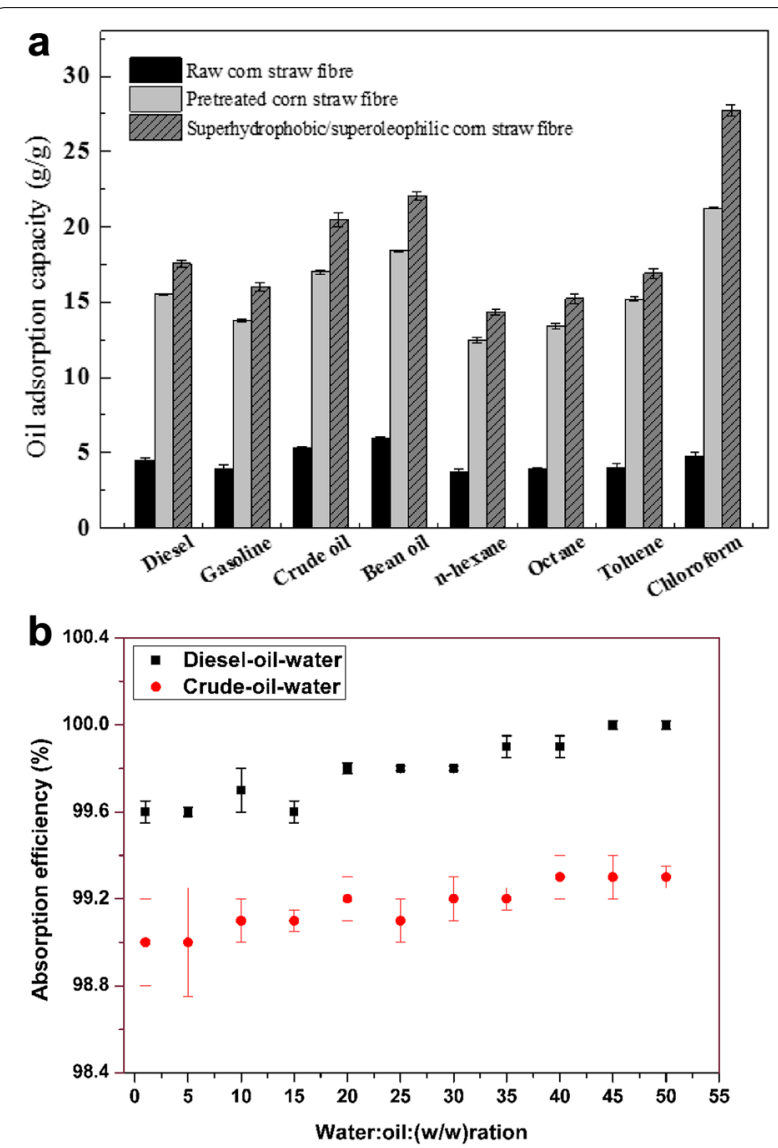

Fig. 8 a The absorption capacities of raw corn straw fiber, pretreated corn straw fiber and prepared superhydrophobic/superoleophilic corn straw fiber for various oils and organic solvents; $\mathbf{b}$ absorption efficiency of superhydrophobic/superoleophilic corn straw with different mass ratios of water to oil 
straw fiber and the prepared superhydrophobic/superoleophilic corn straw fiber in various oils and organic solvents. The adsorption capacity of raw corn straw fiber was very low and was always less than $10 \mathrm{~g} / \mathrm{g}$. In contrast with the raw fiber, the pretreated corn straw fiber exhibited a higher absorption capacity for all oils and organic solvents, with its value almost 3 times higher than pristine corn straw fiber. Moreover, the oil absorption quantity of the prepared superhydrophobic/superoleophilic corn straw fiber for diesel oil, crude oil, bean oil, and chloroform was relatively large at $17.5,20.3,22.6$, and 27.8 times their own quality, respectively; however, the oil absorption quantity of the prepared superhydrophobic/superoleophilic corn straw fiber for gasoline, $n$-hexane, octane, and toluene was relatively small at 15.5, 13.5, 15.1, and 16.3 times their own quality, respectively. The reason is that the oil absorption capacity is relevant to the viscosity and density of the oil products and organic solvents, with a higher viscosity and density resulting in a saturated absorption capacity of prepared corn straw fiber (Guo et al. 2015). For the same oil or organic solvents, the absorption capacity of the prepared superhydrophobic/superoleophilic corn straw fiber was slightly higher than that of the pretreated corn straw fiber, indicating that the superhydrophobic modification can further enhance the oil capacity, which is beneficial for practical application. In addition to the absorption capacity, the absorption efficiency of the prepared corn straw fiber was studied to ascertain the potential of superhydrophobic/superoleophilic corn straw fiber in oil/water separation. In theory, the superhydrophobic/superoleophilic corn straw fibers absorb very little water; however, there are errors in the oil absorption efficiency. When the water content of the oil-water mixture increased, the prepared corn straw fiber absorbed a small amount of water during magnetic stirring. The oil removal efficiency of the resulting fiber for diesel oil and crude oil varied from 100 to $99 \%$, with different mass ratios of water-to-oil (Fig. 8b). The main cause of this phenomenon is that there was a small amount of water absorbed at the same time that the prepared corn straw fibers absorb oil (Wang et al. 2013), which indicates that the prepared fiber can be widely applied for oil removal from water. Taken together, because of the high absorption capacity and oil removal efficiency, we clearly demonstrate that the novel superhydrophobic/superoleophilic corn straw fiber obtained in this study can be regarded as a highefficient oil absorbent with great chemical stability and environmental durability. Moreover, it has a higher oil adsorption capacity, compared to other biomass-based absorbents (Table 1).
Table 1 Comparison of the oil absorption capacity of some recently reported oil sorbent and the samples prepared in this study taking diesel oil for example

\begin{tabular}{|c|c|c|c|}
\hline Oil & Oil sorbent & $\begin{array}{l}\text { Oil adsorption capacity } \\
(\mathbf{g} / \mathbf{g})\end{array}$ & References \\
\hline \multirow[t]{6}{*}{ Diesel } & $\begin{array}{l}\text { Corn straw } \\
\text { fiber }\end{array}$ & 17.5 & This study \\
\hline & Peat sorb & 2.7 & (Ribeiro et al. 2003) \\
\hline & Sugi bark & 16.5 & (Saito et al. 2003) \\
\hline & Cotton & 41 & (Liu et al. 2014) \\
\hline & $\begin{array}{l}\text { Corn straw } \\
\text { fiber }\end{array}$ & 18 & (Zang et al. 2016) \\
\hline & Sawdust & 11.5 & (Gan et al. 2016) \\
\hline
\end{tabular}

\section{Conclusions}

In this study, we successfully developed a preparation process for a novel superhydrophobic/superoleophilic corn straw fiber by attachment of PTES-modified $\mathrm{SiO}_{2}$ particles onto the fiber surface via the sol-gel and impregnation method. The prepared corn straw fiber exhibited outstanding properties of superhydrophobicity and simultaneous superoleophilicity with a water contact angle of $152^{\circ}$ and an oil contact angle of $0^{\circ}$ for different oils. In addition, the microtopography, wetting property, chemical composition and oil absorption performance were comprehensively studied. Results revealed that $\mathrm{SiO}_{2}$ granules successfully modified by PTES were robustly attached to the fiber surface, resulting in a hierarchical structure and low surface energy, thus giving rise to the significant phenomenon of both superhydrophobicity and superoleophilicity. Moreover, the prepared superhydrophobic/superoleophilic corn straw fiber displayed great chemical stability and environmental durability. Most importantly, the prepared superhydrophobic/ superoleophilic corn straw fiber possessed an excellent absorption capacity and high absorption efficiency. Taken together, these results demonstrate that the prepared fiber obtained in this study exhibits a high application potential to effectively separate oil/water mixtures.

\footnotetext{
Abbreviations

PTES: (Heptadecafluoro-1,1,2,2-tetradecyl) trimethoxysilane; FTIR: Fourier transformation infrared spectroscope; XPS: X-ray photoelectron spectroscopy; EDX: energy-dispersive X-ray analysis; WCA: water contact angle; OCA: oil contact angle; SEM: scanning electron microscope.

\section{Equation parameters}

$\gamma_{s v^{\prime}} \gamma_{s l}$ and $\gamma_{l v}$ : solid-vapor, solid-liquid and liquid-vapor interfacial tensions, respectively; $\theta$ : contact angle; $q$ : sorption capability $(\mathrm{g} / \mathrm{g}) ; m_{2}$ : the weight of corn straw fibers after absorption; $m_{1}$ : the initial weight of corn straw fibers before absorption; $k$ : oil removal efficiency $(\%) ; w_{3}$ : the weight of corn straw fibers after absorption; $w_{2}$ : the initial weight of corn straw fibers before absorption; $\boldsymbol{w}_{1}$ : the weight of water absorbed in the absorbents.
} 


\section{Authors' contributions}

$Y X$ and $H Y$ designed the study, performed experiments, analyzed data, and prepared the manuscript. DZ, FL and XH contributed to the discussion. CY, $\mathrm{SHH}$, JSC and YZ reviewed the results, helped in data analysis, and edited the manuscript. All authors read and approved the final manuscript.

\section{Author details}

${ }^{1}$ Key Laboratory of Bio-based Material Science and Technology, Ministry of Education, Northeast Forestry University, Harbin 150040, People's Republic of China. ${ }^{2}$ State Key Laboratory of Urban Water Resource and Environment, School of Environment, Harbin Institute of Technology, Harbin 150090, People's Republic of China. ${ }^{3}$ Department of Chemical Engineering, National Cheng Kung University, Tainan, Taiwan. ${ }^{4}$ President Office, Harbin Medical University, Harbin 150001, People's Republic of China.

\section{Acknowledgements}

The authors would like to acknowledge English-editing support by Paul Steed at NCKU language center.

\section{Competing interests}

The authors declare that they have no competing interests.

\section{Availability of data and materials}

All data generated or analyzed during this study are included in this article.

\section{Consent for publication}

Not applicable.

\section{Ethics approval and consent to participate}

Not applicable.

\section{Funding}

This research was supported by the Fundamental Research Funds for the Central Universities (2572015EB01) and the National Natural Science Foundation of China (31470584).

\section{Publisher's Note}

Springer Nature remains neutral with regard to jurisdictional claims in published maps and institutional affiliations.

Received: 10 October 2017 Accepted: 8 February 2018

Published online: 20 February 2018

\section{References}

Angelova D, Uzunov I, Uzunova S, Gigova A, Minchev L (2011) Kinetics of oil and oil products adsorption by carbonized rice husks. Chem Eng J 172(1):306-311. https://doi.org/10.1016/j.cej.2011.05.114

Autumn K, Liang YA, Hsieh ST, Zesch W, Chan WP, Kenny TW, Fearing R, Full RJ (2000) Adhesive force of a single gecko foot-hair. Nature 405:681. https:// doi.org/10.1038/35015073

Boopathy R, Shields S, Nunna S (2012) Biodegradation of crude oil from the BP oil spill in the marsh sediments of Southeast Louisiana USA. Appl Biochem Biotechnol 167(6):1560-1568. https://doi.org/10.1007/ s12010-012-9603-1

Cojocaru C, Macoveanu M, Cretescu I (2011) Peat-based sorbents for the removal of oil spills from water surface: application of artificial neural network modeling. Colloids Surf A Physicochem Eng Asp 384(1):675-684. https://doi.org/10.1016/j.colsurfa.2011.05.036

Deng D, Prendergast DP, MacFarlane J, Bagatin R, Stellacci F, Gschwend PM (2013) Hydrophobic meshes for oil spill recovery devices. ACS Appl Mater Interfaces 5(3):774-781. https://doi.org/10.1021/am302338x

Gan W, Gao L, Zhang W, Li J, Cai L, Zhan X (2016) Removal of oils from water surface via useful recyclable $\mathrm{CoFe}_{2} \mathrm{O}_{4}$ /sawdust composites under magnetic field. Mater Design 98:194-200. https://doi.org/10.1016/j. matdes.2016.03.018

Gao J, Liu Y, Xu H, Wang Z, Zhang X (2009) Mimicking biological structured surfaces by phase-separation micromolding. Langmuir 25(8):4365-4369. https://doi.org/10.1021/la9008027
Guo P, Zhai S, Xiao Z, An Q (2015) One-step fabrication of highly stable, superhydrophobic composites from controllable and low-cost PMHS/ TEOS sols for efficient oil cleanup. J Colloid Interface Sci. 446(Supplement C):155-162. https://doi.org/10.1016/j.jcis.2015.01.062

Howarter JA, Youngblood JP (2007) Self-cleaning and anti-fog surfaces via stimuli-responsive polymer brushes. Adv Mater 19(22):3838-3843. https://doi.org/10.1002/adma.200700156

Hsieh C-T, Chen W-Y, Wu F-L, Hung W-M (2010) Superhydrophobicity of a three-tier roughened texture of microscale carbon fabrics decorated with silica spheres and carbon nanotubes. Diam Relat Mater 19(1):26-30. https://doi.org/10.1016/j.diamond.2009.10.017

Ju J, Bai H, Zheng Y, Zhao T, Fang R, Jiang L (2012) A multi-structural and multi-functional integrated fog collection system in cactus. Nat Commun 3:1247. https://doi.org/10.1038/ncomms2253

Karakasi OK, Moutsatsou A (2010) Surface modification of high calcium fly ash for its application in oil spill clean up. Fuel 89(12):3966-3970. https://doi. org/10.1016/j.fuel.2010.06.029

Kim H, Noh K, Choi C, Khamwannah J, Villwock D, Jin S (2011) Extreme superomniphobicity of multiwalled $8 \mathrm{~nm} \mathrm{TiO} 2$ nanotubes. Langmuir 27(16):10191-10196. https://doi.org/10.1021/la2014978

Kulkarni SA, Ogale SB, Vijayamohanan KP (2008) Tuning the hydrophobic properties of silica particles by surface silanization using mixed selfassembled monolayers. J Colloid Interface Sci 318(2):372-379. https://doi. org/10.1016/j.jcis.2007.11.012

Li Y, Li L, Sun J (2010) Bioinspired self-healing superhydrophobic coatings. Angew Chem 122(35):6265-6269. https://doi.org/10.1002/ ange.201001258

Liang W, Guo Z (2013) Stable superhydrophobic and superoleophilic soft porous materials for oil/water separation. RSC Adv 3(37):16469-16474. https://doi.org/10.1039/C3RA42442A

Liu F, Ma M, Zang D, Gao Z, Wang C (2014) Fabrication of superhydropho$\mathrm{bic} / \mathrm{superoleophilic} \mathrm{cotton} \mathrm{for} \mathrm{application} \mathrm{in} \mathrm{the} \mathrm{field} \mathrm{of} \mathrm{water/oil}$ separation. Carbohyd Polym 103:480-487. https://doi.org/10.1016/j. carbpol.2013.12.022

Ribeiro TH, Rubio J, Smith RW (2003) A dried hydrophobic aquaphyte as an oil filter for oil/water emulsions. Spill Sci Technol B 8(5-6):483-489. https:// doi.org/10.1016/S1353-2561(03)00130-0

Saito M, Ishii N, Ogura S, Maemura S, Suzuki H (2003) Development and water tank tests of sugi bark sorbent (SBS). Spill Sci Technol B 8(5-6):475-482

Schaum J, Cohen M, Perry S, Artz R, Draxler R, Frithsen JB, Heist D, Lorber M, Phillips $L$ (2010) Screening level assessment of risks due to dioxin emissions from burning oil from the BP deepwater horizon Gulf of Mexico spill. Environ Sci Technol 44(24):9383-9389. https://doi.org/10.1021/ es103559w

Vinogradova E, Estrada M, Moreno A (2006) Colloidal aggregation phenomena: spatial structuring of TEOS-derived silica aerogels. J Colloid Interface Sci 298(1):209-212. https://doi.org/10.1016/j.jcis.2005.11.064

Vlaev L, Petkov P, Dimitrov A, Genieva S (2011) Cleanup of water polluted with crude oil or diesel fuel using rice husks ash. J Taiwan Inst Chem Eng 42(6):957-964. https://doi.org/10.1016/j.jtice.2011.04.004

Wang L, Yang S, Wang J, Wang C, Chen L (2011a) Fabrication of superhydrophobic TPU film for oil-water separation based on electrospinning route. Mater Lett 65(5):869-872. https://doi.org/10.1016/j.matlet.2010.12.024

Wang S, Liu C, Liu G, Zhang M, Li J, Wang C (2011 b) Fabrication of superhydrophobic wood surface by a sol-gel process. Appl Surf Sci 258(2):806-810. https://doi.org/10.1016/j.apsusc.2011.08.100

Wang B, Li J, Wang G, Liang W, Zhang Y, Shi L, Guo Z, Liu W (2013) Methodology for robust superhydrophobic fabrics and sponges from in situ growth of transition metal/metal oxide nanocrystals with thiol modification and their applications in oil/water separation. ACS Appl Mater Interfaces 5(5):1827-1839. https://doi.org/10.1021/am303176a

Wang S, Liu K, Yao X, Jiang L (2015) Bioinspired surfaces with superwettability: new insight on theory, design, and applications. Chem Rev 115(16):82308293. https://doi.org/10.1021/cr400083y

Wei ZJ, Liu WL, Tian D, Xiao CL, Wang XQ (2010) Preparation of lotus-like superhydrophobic fluoropolymer films. Appl Surf Sci 256(12):3972-3976. https://doi.org/10.1016/j.apsusc.2010.01.059

Yao X, Song Y, Jiang L (2011) Applications of bio-inspired special wettable surfaces. Adv Mater 23(6):719-734. https://doi.org/10.1002/adma.201002689

Yuan Z, Wang X, Bin J, Peng C, Xing S, Wang M, Xiao J, Zeng J, Xie Y, Xiao X, Fu X, Gong H, Zhao D (2013) A novel fabrication of a superhydrophobic 
surface with highly similar hierarchical structure of the lotus leaf on a copper sheet. Appl Surf Sci 285(Part B):205-210. https://doi.org/10.1016/j. apsusc.2013.08.037

Zang D, Liu F, Zhang M, Niu X, Gao Z, Wang C (2015) Superhydrophobic coating on fiberglass cloth for selective removal of oil from water. Chem Eng $J$ 262:210-216. https://doi.org/10.1016/j.cej.2014.09.082

Zang D, Zhang M, Liu F, Wang C (2016) Superhydrophobic/superoleophilic corn straw fibers as effective oil sorbents for the recovery of spilled oil. J Chem Technol Biotechnol 91(9):2449-2456. https://doi.org/10.1002/ jctb.4834

Zhang Y, Wang H, Yan B, Zhang Y, Yin P, Shen G, Yu R (2008) A rapid and efficient strategy for creating super-hydrophobic coatings on various material substrates. J Mater Chem 18(37):4442-4449. https://doi.org/10.1039/ B801212A
Zhang W, Shi Z, Zhang F, Liu X, Jin J, Jiang L (2013) Superhydrophobic and superoleophilic PVDF membranes for effective separation of water-inoil emulsions with high flux. Adv Mater 25(14):2071-2076. https://doi. org/10.1002/adma.201204520

Zhou X, Zhang Z, Xu X, Guo F, Zhu X, Men X, Ge B (2013) Robust and durable superhydrophobic cotton fabrics for oil/water separation. ACS Appl Mater Interfaces 5(15):7208-7214. https://doi.org/10.1021/am4015346

\section{Submit your manuscript to a SpringerOpen ${ }^{\circ}$ journal and benefit from:}

- Convenient online submission

- Rigorous peer review

- Open access: articles freely available online

- High visibility within the field

- Retaining the copyright to your article

Submit your next manuscript at $\boldsymbol{\nabla}$ springeropen.com 